\title{
IgG4-Related Pancreatitis: A Case Report and Review of Literature
}

\author{
Chunlin Li ${ }^{1,2}$, Jin Huang1,3, Boxu Ren ${ }^{1,2 *}$ \\ ${ }^{1}$ Department of Medical Imaging, Medical School of Yangtze University, Jingzhou, China \\ ${ }^{2}$ Department of Intervention Radiology, The Second Clinical College of Yangtze University, Jingzhou Central Hospital, Jingzhou, China \\ ${ }^{3}$ Laifeng People's Hospital, Enshi, China \\ Email: ^boxuren188@163.com, ^244806621@qq.com
}

How to cite this paper: Li, C.L., Huang, J. and Ren, B.X. (2020) IgG4-Related Pancreatitis: A Case Report and Review of Literature. Yangtze Medicine, 4, 310-318. https://doi.org/10.4236/ym.2020.44029

Received: December 22, 2019

Accepted: September 27, 2020

Published: September 30, 2020

Copyright () 2020 by author(s) and Scientific Research Publishing Inc. This work is licensed under the Creative Commons Attribution International License (CC BY 4.0).

http://creativecommons.org/licenses/by/4.0/ (c) (i) Open Access

\begin{abstract}
Background: Immunoglobulin G4-related pancreatitis (IgG4-RAIP) is a relatively rare chronic inflammatory disease of the pancreas. The cause of this disease is still unclear. Due to the lack of specific clinical symptoms and imaging findings, it is often misdiagnosed as pancreatic cancer. Clinicians are paying more and more attention to it. Case report: We are reporting an IgG4-RAIP case, in which the patient had been misdiagnosed as pancreatic cancer and prepared for surgical treatment but finally was diagnosed as IgG4-RAIP after pancreatic biopsy and serum immune test. And the patient was recovered after the treatment of glucocorticoids. Discussion: In order to make more patients get correct diagnosis and reasonable treatment, when we encounter undiagnosed cases of pancreatitis or pancreatic cancer in clinical work, it is very important to conduct pancreatic biopsy and serum immune blood test before operation for these patients. Conclusion: Pancreatic biopsy and serum immune blood test can effectively reduce the occurrence of misdiagnosis of IgG4-RAIP.
\end{abstract}

\section{Keywords}

IgG4, IgG4-RAIP, Pancreatitis, Pancreatic Cancer, Glucocorticoid

\section{Introduction}

The concept of autoimmune pancreatitis (AIP) was first put forward by Yoshida et al. in 1995 [1]. At present, it is internationally recognized that the disease is divided into two types, namely type 1 and type 2 , in which type 1 is more common, or called immunoglobulin G4-related pancreas (IgG4-RAIP), type 2 is rel- 
atively rare [2]. A study by Japanese scholar Okazaki $\mathrm{K}$ et al. showed that type 1 is characterized by elevated serum IgG4 and is often associated with extrapancreatic tissue lesions, especially in 50- to 70-year-old males [3]. Another global epidemiological survey showed that the average age of the first diagnosis of IgG4-RAIP was 61.4 years [4]. Type 2 pancreatitis, also known as idiopathic central ductal pancreatitis, is mainly characterized by granulocyte epithelial damage in pancreatic tissue; serum IgG4 is generally not elevated, rarely involving extra-pancreatic tissue; the age of onset is usually lower than type 1; this type of clinical is rare. The etiology and pathogenesis of IgG4-RAIP have not been fully elucidated [5] [6]. At present, most scholars believe that the disease is a disease with the participation of multiple factors and mechanisms, in which the immune mechanism is dominant, that is, pancreatic diseases mediated by autoimmune mechanisms, mainly cytoplasmic and lymphocyte infiltration, are often accompanied by pancreatic fibrosis and pancreatic dysfunction. It is a rare chronic inflammatory disease of the pancreas [7]. The onset of the disease is usually concealed; painless obstructive jaundice is the first symptom for most patients, accounting for about $75 \%$ [8]; some patients may be accompanied by mid-upper abdominal discomfort or mild to moderate pain [9]. Imaging examination is often localized or diffuses enlargement of the pancreas as the main manifestation, so it is often misdiagnosed as pancreatic cancer, and the misdiagnosis rate is as high as $56 \%$ [10] [11]. The case reported in this article has also been misdiagnosed as pancreatic cancer and even nearly underwent surgical treatment. The purpose of this report is to summarize the standardized diagnosis and treatment of IgG4-RAIP, provide clinical reference, reduce the misdiagnosis rate, reduce unnecessary surgical treatment, and improve the prognosis of patients.

\section{Case History}

A 74-year-old male patient was admitted to hospital because of "pancreatic mass found for more than one month". The patient was treated with pancreatitis in another hospital 2 years ago because of epigastric pain. After discharge, the pain of epigastrium and xiphoid process appeared repeatedly, and the effect of symptomatic treatment was not good. A month ago, the epigastric pain occurred again, and there was no improvement in symptomatic treatment. The abdominal computed tomography (CT) examination in the outpatient clinic of our hospital showed pancreatic mass and choledochal dilatation. Therefore, he was incorporated into our department with "pancreatic space occupying with choledochal dilatation" and planned to undergo surgical resection of "pancreatic tumor".

\section{Examination, Diagnosis, Differential Diagnosis and Treatment}

The values of cancer antigen 12-5 (CA12-5), CA19-9, alpha-fetoprotein test (AFP) and carcinoembryonic antigen (CEA) in blood examination were all within the normal range. Plasma D-dimer (308 ng/ml), blood amylase (283.5 
$\mathrm{U} / \mathrm{L})$ and jaundice index $(+)$ were increased. Color Doppler ultrasonography revealed enlargement of the gallbladder with cholestasis, dilatation of intrahepatic bile duct and pancreatic duct, and abnormal echo in the head of the pancreas (space-occupying lesions to be excluded) (Figure 1(a)). Magnetic resonance imaging (MRI) plain scan showed nodular mass in the head of pancreas with slightly decreased T1-weighted imaging (T1WI) signal and slightly increased T2-weighted imaging (T2WI) signal (Figures 1(b)-(d)); Magnetic resonance cholangiopancreatography (MRCP) showed mass in the head of pancreas; truncation of the head of common bile duct and pancreas, dilatation of intrahepatic bile duct, common bile duct and pancreatic duct, enlargement of gallbladder (Figure 1(h)). Enhanced CT scan of the upper abdomen showed small patches of slightly low-density shadow in the head of the pancreas, unclear boundaries, edge enhancement in the arterial phase, and filling enhancement gradually in the venous phase and delayed phase (Figures $1(\mathrm{e})-(\mathrm{h})$ ). Combined with the above imaging and biochemical examination, it was diagnosed as cancer of the head of the pancreas with obstructive jaundice. Percutaneous transhepatic cholangial drainage (PTCD) was performed to relieve the symptoms of biliary obstruction. In order to further confirm whether the diagnosis was correct, pancreatic tumor biopsy was performed under the guidance of CT 4 days later. Cytological hematoxylin-eosin (HE) staining showed that a large number of lymphocytes and some necrotic cells were seen under the microscope, and a few enlarged naked nuclei were seen (Figure 2(a) and Figure 2(b)). Pathological examination showed that interlobular fibrous tissue hyperplasia with a large number of

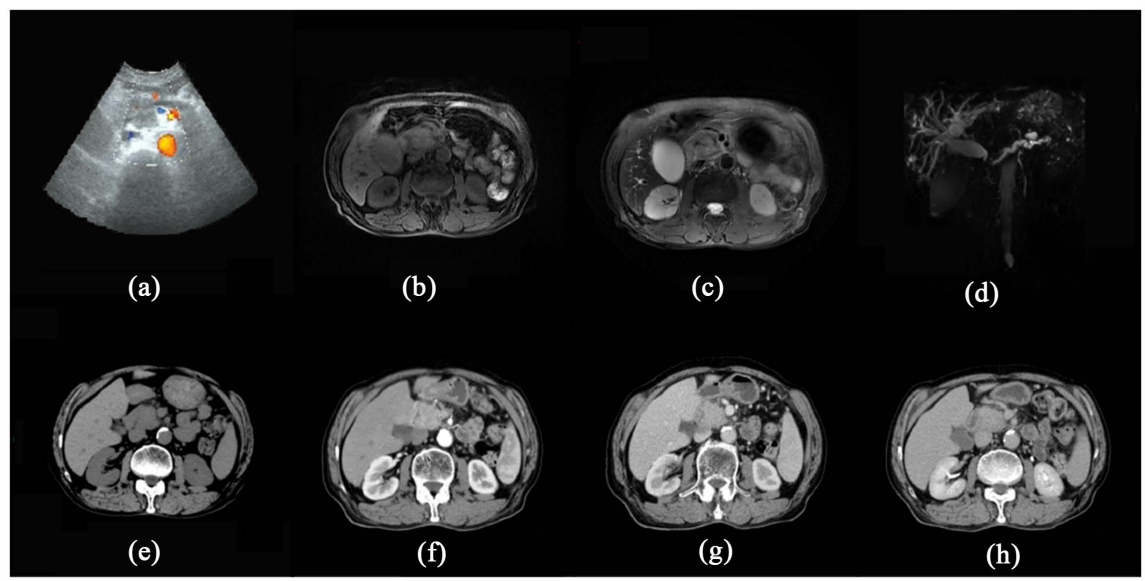

Figure 1. (a) Color doppler ultrasonography showed that slightly hypoechoic shadow could be seen in the pancreatic head area, the boundary was not clear, and there was no obvious blood flow signal in the lesion area. (b)-(d) MRI and MRCP: the pancreatic head area enlarged, the signal intensity of T1WI sequence decreased slightly, the signal intensity of T2WI sequence increased slightly, and MRCP showed dilatation of intrahepatic and extrahepatic bile duct and pancreatic duct. (e)-(h) CT examination: on plain scan, the pancreatic head area was enlarged, nodular isodensity shadow was seen, the boundary was unclear, and the pancreatic duct was dilated; enhanced scan showed slight enhancement of the pancreatic head area in the arterial phase and filling enhancement in the venous phase and delayed phase. 


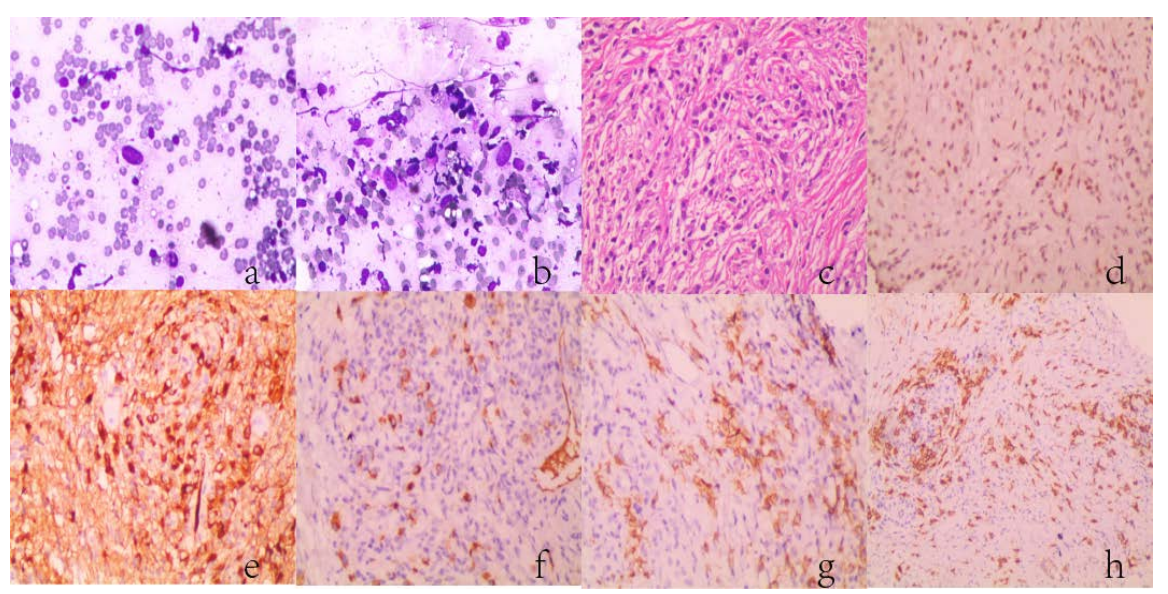

Figure 2. (a) (b) Cytological examination: a large number of lymphocytes and some necrotic cells were seen under the microscope, and a large number of enlarged naked nuclei were seen; (c) (d) Pathological HE staining: microscopically, diffuse interlobular fibrous tissue hyperplasia with a large number of chronic inflammatory cell infiltration, mainly lymphocytes and plasma cells, a small amount of chronic inflammatory cell infiltration could be seen in the lobules, and slight acinar hyperplasia could be seen in some areas; (e) (f) Pathological immunohistochemistry: plasma cells were positive for CD38, CD138, IgG, and partially positive for IgG4, in which IgG4 > $10 \mathrm{HPF}$, and IgG4/IgG > 40\% Bing p53 negative.

chronic inflammatory cell infiltration, mainly lymphocytes and plasma cells, a small amount of chronic inflammatory cells were seen in the lobules, slight acinar hyperplasia could be seen in some areas, and no other special abnormal changes were seen (Figure 2(c) and Figure 2(d)) [12]. The pathological examination showed that pancreatic interlobular fibrous tissue hyperplasia with a large number of chronic inflammatory cell infiltration, mainly lymphocytes and plasma cells. Because no tumor cells were found in pathological examination, there were mainly chronic inflammatory changes. Combined with the indexes of imaging and biochemical examination, we believe that it may be an autoimmune-related disease. The biopsy specimens were examined by immunohistochemistry again. The results showed that the plasma cells were positive for CD38, CD138 and IgG, and partially positive for IgG4, in which IgG4 > 10/HPF and IgG4/IgG > 40\%. Serum immunoglobulin $\mathrm{A}$ and immunoglobulin $\mathrm{M}$ were normal, but immunoglobulin $\mathrm{G}$ was elevated $(19.31 \mathrm{~g} / \mathrm{L})$ and antinuclear antibody was positive $(+)$. It is consistent with the changes of IgG4-RAIP (Figures 2(e)-(h)). The serum IgG4 level increased on the next day $(13 \mathrm{~g} / \mathrm{L})$. Reviewing this patient's recurrent symptoms of epigastric pain in the past two years, combined with the results of all imaging, biochemical and pathological examinations after admission, we finally diagnosed IgG4-RAIP and sclerosing cholangitis. Therefore, we decided to treat the patient with prednisone, $40 \mathrm{mg} / \mathrm{day}$, oral administration [13].

\section{Results and Follow-Up}

After 4 weeks of oral glucocorticoid treatment, plain CT scan and enhanced MRI examination showed that the lesion was significantly reduced (Figure 3 ), and the 


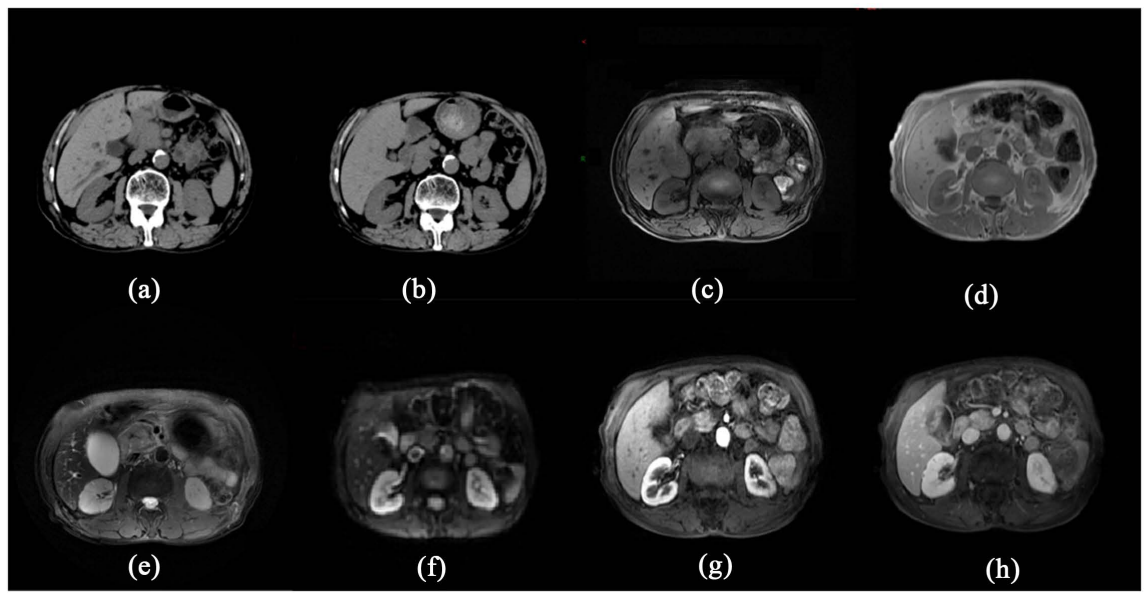

Figure 3. (a) Pre-treatment CT plain scan; (b) Plain scan of CT after 4 weeks of treatment; (c) Pre-treatment T1WI; (d) T1WI after 4 weeks treatment; (e) Pre-treatment T2WI; (f) T2WI after 4 weeks treatment; They showed that the size of the focus was reduced after treatment, the T1WI signal of the focus was higher than that before treatment, and the signal intensity of T2WI was slightly lower than that before treatment; (g) (h) After 4 weeks of treatment, MRI enhancement showed that the lesions showed filling enhancement.

symptoms of epigastric pain were completely disappeared. We will adhere to the long-term follow-up of the patient to observe the medium-and long-term effect.

\section{Discussion}

In this case, pancreatic mass was found by imaging examination, but eventually was diagnosed as IgG4-RAIP, and the patient had a good effect on glucocorticoid treatment. At present, it is believed that IgG4-RAIP can affect all organs of the human body, not only for a single organ, but also for multiple organs at the same time, and the pancreas is one of the most frequently affected organs [14]. After many efforts, research and comprehensive evaluation, the International Association of Pancreatic Diseases finally released the diagnostic criteria of IgG4-RAIP in 2011, which mainly includes five basic features: 1) Imaging changes of pancreatic parenchyma or pancreatic duct; 2) Serum IgG4 level; 3) Involvement of other organs outside the pancreas; 4) Histopathological findings of pancreas; 5) Sensitivity to glucocorticoid therapy (2). The imaging findings of IgG4-RAIP can be divided into three types: diffuse type, focal type and multifocal type [15]. Among them, diffuse type is the most common, imaging examination showed diffuse enlargement of pancreas; CT showed decreased density of pancreas in the lesion area, MRI showed decreased T1WI signal in the lesion area, T2WI signal increased slightly, the internal structure of pancreatic parenchyma disappeared, and the edge was clear. Usually, the pancreas showed "sausage-like" changes and the fat space around the pancreas was clear [16]. CT or MRI enhanced lesions showed signs of progressive enhancement, that is, slight enhancement in arterial phase, and gradual increase in enhancement in venous phase and delayed phase. About $36 \%$ of patients showed characteristic "halo 
sign". The localized type usually occurred in the head of pancreas, and the ultrasonography showed hypoechoic lesions in the focus area [17]; CT or MRI showed focal low-density lesions or decreased signal intensity on T1WI, localized enlargement of the pancreas and mild dilatation of the pancreatic duct upstream of the focus. MRCP showed dilatation of intrahepatic bile duct, truncation of the head of common bile duct and pancreas and dilatation of pancreatic duct. This type is easy to be confused with pancreatic cancer [18]. Contrast-enhanced CT showed enlargement of the head of pancreas, dilatation of pancreatic duct, obvious enhancement at the edge of the focus in arterial phase, filling-like delayed enhancement in venous phase and delayed phase (Figures 1(b)-(e)); focal hypoechoic area, dilatation of intrahepatic and extrahepatic bile duct and pancreatic duct were vaguely seen in color Doppler ultrasonography (Figure 1(a)). It is consistent with the imaging findings of localized IgG4-RAIP reported in the literature. An international multicenter study shows that the sensitivity and specificity of endoscopic retrograde cholangiopancreatography (ERCP) in the diagnosis of IgG4-associated pancreatitis are $71 \%$ and $83 \%$ respectively [19]. Because our hospital does not have the conditions for ERCP, this test is not performed. In terms of serological examination, the most valuable indicator of IgG4-RAIP is the increase of serum IgG4 [20], and more than 2 times higher than normal serum IgG4 can significantly increase the accuracy of diagnosis, with a sensitivity and specificity of $93 \%$ and $99 \%$, respectively. A small number of patients with IgG4-associated pancreatitis may be associated with slightly elevated CA19-9 [21] [22]. In this case, the level of serum IgG4 was 13 g/L, while CA19-9 was normal.

IgG4-RAIP is often accompanied by involvement of other organs outside the pancreas, among which sclerosing cholangitis and retroperitoneal fibrosis are the most common (3). According to the guidelines of the International Association of Pancreatology on autoimmune pancreatitis (2) and studies on the clinicopathological characteristics of IgG4-related diseases (IgG4-RD) [23], histopathological changes of IgG4-related pancreatitis include: 1) there are a large number of lymphocytes, granulocytes and plasmacytes around the pancreatic duct, showing interstitial inflammatory changes or interlobular fibrosis; 2) the number of IgG4-positive plasma cells > 10/HFP, IgG4/IgG4 > 40\%; 3) pancreatic parenchyma showed striated fibrosis; 4) occlusive venous inflammation. According to these criteria, in the diagnosis of IgG4-RD, the sensitivity can reach $84.9 \%$, the specificity can reach $100 \%$, and the accuracy can reach $93.8 \%$ [24].

IgG4-RAIP and cancer of the head of pancreas have many characteristics in common $(10,11)$. However, the treatment of each disease is very different. Glucocorticoid is the first choice for the treatment of IgG4-RAIP. A multicenter cohort study by Ebbo $\mathrm{M}$ et al. shows that more than $90 \%$ of patients are sensitive to hormone therapy, and most patients can significantly alleviate their clinical symptoms after 2 weeks of hormone treatment, and imaging and serological tests can be significantly improved [25]. There is no international consensus on the initial dose and maintenance dose of hormone therapy for the disease. At 
present, it is recommended to take prednisone orally for $30 \mathrm{mg}-40 \mathrm{mg} /$ days or $0.6 \mathrm{mg} / \mathrm{kg} /$ days for 4 weeks, then gradually reduce the dose according to the patient's clinical symptoms, imaging signs and serological examination indicators, and then maintain the drug dose for $5 \mathrm{mg} /$ days for more than half a year. After half a year of maintenance treatment, if the patient's clinical symptoms disappear. The normal size and shape of pancreas by imaging examination and normal IgG4 by serological examination can be used as indications for drug withdrawal [26].

\section{Conclusion}

IgG4-RAIP is easily misdiagnosed as acute/chronic pancreatitis or pancreatic cancer due to the lack of specific clinical symptoms and special imaging findings, thus delaying the treatment of patients or leading to wrong treatment, and even resulting in unnecessary surgical treatment. Therefore, when we encounter patients with suspected IgG4-RAIP or patients with acute/chronic pancreatitis or pancreatic cancer who cannot be completely diagnosed, we need systematic imaging and serological examinations. For those who still have doubts about serum immunological examination, targeted puncture biopsy is recommended, and when the conditions for biopsy are not available, corticosteroids can be used for diagnostic treatment [11] [27].

\section{Ethical Approval and Consent to Participants}

The involved patient has signed the consent form for this case report.

\section{Funding}

National Natural Science Foundation of China: No. 81772223.

\section{Authors' Contributions}

LCL and JH contributed equally.

Authors contributed to this paper with the design (LCL, JH, and BXR), literature search (LCL and JH), drafting (LCL, JH), revision (JH and BXR), editing (LCL and JH) and final approval (LCL, JH and BXR).

\section{Conflicts of Interest}

The authors declare no conflicts of interest regarding the publication of this paper.

\section{References}

[1] Yoshida, K., Toki, F., Takeuchi, T., et al. (1995) Chronic Pancreatitis Caused by an Autoimmune Abnormality. Proposal of the Concept of Autoimmune Pancreatitis. Digestive Diseases and Sciences, 40, 1561-1568. https://doi.org/10.1007/BF02285209

[2] Shimosegawa, T., Chari, S.T., Frulloni, L., et al. (2011) International Consensus Diagnostic Criteria for Autoimmune Pancreatitis: Guidelines of the International As- 
sociation of Pancreatology. Pancreas, 40, 352-358. https://doi.org/10.1097/MPA.0b013e3182142fd2

[3] Okazaki, K., Uchida, K., Miyoshi, H., et al. (2011) Recent Concepts of Autoimmune Pancreatitis and IgG4-Related Disease. Clinical Reviews in Allergy \& Immunology, 41, 126-138. https://doi.org/10.1007/s12016-010-8214-2

[4] Hart, P.A., Kamisawa, T., Brugge, W.R., et al. (2013) Long-Term Outcomes of Autoimmune Pancreatitis: A Multicentre, International Analysis. Gut, 62, 1771-1776. https://doi.org/10.1136/gutjnl-2012-303617

[5] Huang, B.Y., Wang, Q.X. and Ma, X. (2018) Pathogenesis of Immunoglobulin G4-Related Hepatobiliary Disease. Chinese Journal of Hepatology, 26, 472-475.

[6] Okazaki, K. and Uchida, K. (2018) Current Concept of Autoimmune Pancreatitis and IgG4-Related Disease. The American Journal of Gastroenterology, 113, 1412-1416. https://doi.org/10.1038/s41395-018-0184-7

[7] Palazzo, E., Palazzo, C. and Palazzo, M. (2014) IgG4-Related Disease. Joint, Bone, Spine: Revue du rhumatisme, 81, 27-31. https://doi.org/10.1016/j.jbspin.2013.06.001

[8] Kamisawa, T., Chari, S.T., Giday, S.A., et al. (2011) Clinical Profile of Autoimmune Pancreatitis and Its Histological Subtypes: An International Multicenter Survey. Pancreas, 40, 809-814. https://doi.org/10.1097/MPA.0b013e3182258a15

[9] López-Serrano, A., Crespo, J., Pascual, I., et al. (2016) Diagnosis, Treatment and Long-Term Outcomes of Autoimmune Pancreatitis in Spain Based on the International Consensus Diagnostic Criteria: A Multi-Centre Study. Pancreatology. Official Journal of the International Association of Pancreatology, 16, 382-390.

https://doi.org/10.1016/j.pan.2016.02.006

[10] Enjuto Martínez, D.T., Herrera Merino, N., Pérez González, M., et al. (2017) Autoinmune Pancreatitis: Differential Diagnosis with Pancreatic Adenocarcinoma. Cirugia Espanola, 95, 480-482. https://doi.org/10.1016/j.ciresp.2016.11.005

[11] Dite, P., Novotny, I., Dvorackova, J., et al. (2019) Pancreatic Solid Focal Lesions: Differential Diagnosis between Autoimmune Pancreatitis and Pancreatic Cancer. Digestive Diseases (Basel, Switzerland), 37, 416-421. https://doi.org/10.1159/000499762

[12] Brito-Zerón, P., Bosch, X., Ramos-Casals, M., et al. (2016) IgG4-Related Disease: Advances in the Diagnosis and Treatment. Best Practice \& Research Clinical Rheumatology, 30, 261-278. https://doi.org/10.1016/j.berh.2016.07.003

[13] Haldar, D., Cockwell, P., Richter, A.G., et al. (2016) An Overview of the Diagnosis and Management of Immunoglobulin G4-Related Disease. CMAJ: Canadian Medical Association Journal, 188, 953-961. https://doi.org/10.1503/cmaj.151402

[14] Al-Khalili, O.M. and Erickson, A.R. (2018) IgG-4 Related Disease: An Introduction. Missouri Medicine, 115, 253-256.

[15] Crosara, S., D’Onofrio, M., De Robertis, R., et al. (2014) Autoimmune Pancreatitis: Multimodality Non-Invasive Imaging Diagnosis. World Journal of Gastroenterology, 20, 16881-16890. https://doi.org/10.3748/wjg.v20.i45.16881

[16] Miyoshi, H., Kano, M., Kobayashi, S., et al. (2019) Diffuse Pancreatic Cancer Mimicking Autoimmune Pancreatitis. Internal Medicine, 58, 2523-2527. https://doi.org/10.2169/internalmedicine.2689-19

[17] Su, L.Y., Gao, Y., Xu, G., et al. (2012) Retrospective Analysis of Ultrasonography for Autoimmune Pancreatitis. Chinese Medical Journal, 92, 2649-2651.

[18] Lee, L.K. and Sahani, D.V. (2014) Autoimmune Pancreatitis in the Context of IgG4-Related Disease: Review of Imaging Findings. World Journal of Gastroenter- 
ology, 20, 15177-15189. https://doi.org/10.3748/wjg.v20.i41.15177

[19] Sugumar, A., Levy, M.J., Kamisawa, T., et al. (2011) Endoscopic Retrograde Pancreatography Criteria to Diagnose Autoimmune Pancreatitis: An International Multicentre Study. Gut, 60, 666-670. https://doi.org/10.1136/gut.2010.207951

[20] Choi, E.K., Kim, M.H., Lee, T.Y., et al. (2007) The Sensitivity and Specificity of Serum Immunoglobulin G and Immunoglobulin G4 Levels in the Diagnosis of Autoimmune Chronic Pancreatitis: Korean Experience. Pancreas, 35, 156-161. https://doi.org/10.1097/MPA.0b013e318053eacc

[21] Ghazale, A., Chari, S.T., Smyrk, T.C., et al. (2007) Value of Serum IgG4 in the Diagnosis of Autoimmune Pancreatitis and in Distinguishing It from Pancreatic Cancer. The American Journal of Gastroenterology, 102, 1646-1653. https://doi.org/10.1111/j.1572-0241.2007.01264.x

[22] Ngwa, T., Law, R., Hart, P., et al. (2015) Serum IgG4 Elevation in Pancreatic Cancer: Diagnostic and Prognostic Significance and Association with Autoimmune Pancreatitis. Pancreas, 44, 557-560. https://doi.org/10.1097/MPA.0000000000000297

[23] Detlefsen, S. (2019) IgG4-Related Disease: Microscopic Diagnosis and Differential Diagnosis. Der Pathologe, 40, 619-626. https://doi.org/10.1007/s00292-019-00685-8

[24] Helmberger, T. (2016) Autoimmune Pancreatitis: An Update. Der Radiologe, 56 363-370. https://doi.org/10.1007/s00117-016-0096-8

[25] Ebbo, M., Daniel, L., Pavic, M., et al. (2012) IgG4-Related Systemic Disease: Features and Treatment Response in a French Cohort: Results of a Multicenter Registry. Medicine, 91, 49-56. https://doi.org/10.1097/MD.0b013e3182433d77

[26] Okazaki, K., Chari, S.T., Frulloni, L., et al. (2017) International Consensus for the Treatment of Autoimmune Pancreatitis. Pancreatology: Official Journal of the International Association of Pancreatology, 17, 1-6. https://doi.org/10.1016/j.pan.2016.12.003

[27] Hedfi, M., Charfi, M., Nejib, F.Z., et al. (2019) Focal Mass-Forming Autoimmune Pancreatitis Mimicking Pancreatic Cancer: Which Strategy? La Tunisie Medicale, 97, 731-735. 